\title{
MNK1 pathway activity maintains protein synthesis in rapalog-treated gliomas
}

\author{
Michal Grzmil, ${ }^{1}$ Roland M. Huber, ${ }^{1}$ Daniel Hess, ${ }^{1}$ Stephan Frank, ${ }^{2}$ Debby Hynx, ${ }^{1}$ \\ Gerald Moncayo, ${ }^{1}$ Dominique Klein, ${ }^{1}$ Adrian Merlo, ${ }^{3}$ and Brian A. Hemmings ${ }^{1}$ \\ ${ }^{1}$ Friedrich Miescher Institute for Biomedical Research, Basel, Switzerland. \\ ${ }^{2}$ Division of Neuropathology, Institute of Pathology, University of Basel, Basel, Switzerland. \\ ${ }^{3}$ Neurosurgery and Brain Tumor Research, Bern, Switzerland.
}

\begin{abstract}
High levels of mammalian target of rapamycin complex 1 (mTORC1) activity in malignant gliomas promote tumor progression, suggesting that targeting mTORC1 has potential as a therapeutic strategy. Remarkably, clinical trials in patients with glioma revealed that rapamycin analogs (rapalogs) have limited efficacy, indicating activation of resistance mechanisms. Targeted depletion of MAPK-interacting Ser/Thr kinase 1 (MNK1) sensitizes glioma cells to the mTORC1 inhibitor rapamycin through an indistinct mechanism. Here, we analyzed how MNK1 and $\mathrm{mTORC} 1$ signaling pathways regulate the assembly of translation initiation complexes, using the cap analog $\mathrm{m}^{7}$ GTP to enrich for initiation complexes in glioma cells followed by mass spectrometry-based quantitative proteomics. Association of eukaryotic translation initiation factor 4E (eIF4E) with eIF4E-binding protein 1 (4EBP1) was regulated by the mTORC1 pathway, whereas pharmacological blocking of MNK activity by CGP57380 or MNK1 knockdown, along with mTORC1 inhibition by RAD001, increased 4EBP1 binding to eIF4E. Furthermore, combined MNK1 and mTORC1 inhibition profoundly inhibited 4EBP1 phosphorylation at Ser65, protein synthesis and proliferation in glioma cells, and reduced tumor growth in an orthotopic glioblastoma (GBM) mouse model. Immunohistochemical analysis of GBM samples revealed increased 4EBP1 phosphorylation. Taken together, our data indicate that rapalog-activated MNK1 signaling promotes glioma growth through regulation of 4EBP1 and indicate a molecular cross-talk between the mTORC1 and MNK1 pathways that has potential to be exploited therapeutically.
\end{abstract}

\section{Introduction}

In cancer, deregulated signaling networks influence protein synthesis, promoting disease progression and cancer cell survival. Mammalian target of rapamycin complex 1 (mTORC1), a major regulator of translation, is hyperactivated in brain tumors, including glioblastoma (GBM), the most aggressive form of glioma, with a mean survival of approximately 1 year. Overexpressed or mutated and constitutively active tyrosine receptor kinases activate the phosphatidylinositol-3-OH kinase (PI3K) and AKT signaling pathways that in turn regulate mTORC1 $(1,2)$. In an early preclinical study, mTORC1 inactivation by rapamycin showed promising antiglioma activity in vivo (3), yet clinical trials found only a very limited response of patients with glioma to rapamycin analogs (rapalogs) $(4,5)$ indicating the activation of resistance mechanisms to overcome mTORC1 inhibition.

Activated mTORC1 is composed of mTOR kinase, the adaptor protein raptor, and $\mathrm{mLST} 8$ and supports protein synthesis by the phosphorylation of eukaryotic translation initiation factor 4E-binding (eIF4E-binding) proteins (4EBPs), translation initiation factor scaffold protein eIF4G, and S6 kinase (S6K), as reviewed recently (6-8). Active S6K phosphorylates ribosomal protein S6 and RNA helicase cofactor eIF4B and inactivates eEF2 kinase $(\mathrm{eEF} 2 \mathrm{~K})$, which inhibits elongation factor eEF2 as well as translation inhibitor programmed cell death 4 (PDCD4) by inducing its proteasomal degradation. Notably, mTORC1 influences the abundance of translation initiation factor eIF4E, the cap-binding protein that limits cap-dependent translation. Non-

Conflict of interest: The authors have declared that no conflict of interest exists. Citation for this article: J Clin Invest. 2014;124(2):742-754. doi:10.1172/JCI70198 phosphorylated 4EBPs interact with and sequester eIF4E, resulting in decreased formation of the eIF4F complex of eIF4E, eIF4G, and 5'UTR unwinding RNA helicase eIF4A. Phosphorylation of 4EBPs by mTORC1 leads to eIF4E release and assembly of eIF4F, which associates with mRNA at its 5'UTR during cap-dependent translation initiation. Overexpression of eIF4E causes oncogenic transformation (9), and increased eIF4E protein levels are found in the majority of human cancers, in which they are correlated with poor prognosis (10). Thus, eIF4E oncogenic activity is a potential therapeutic drug target.

During translation initiation, eIF4E activity can also be modified by MAPK-interacting kinases MNK1 and MNK2, which bind to translation initiation complexes via eIF4G and phosphorylate eIF4E at Ser209 (11). It has been shown that eIF4E phosphorylation by MNKs is decisive for the eIF4E activity that opposes apoptosis and promotes tumorigenesis in vivo and that overexpression of constitutively active MNK1 has an oncogenic effect similar to that of eIF4E (12). Phosphorylation of eIF4E is enhanced in many human cancers (13), and the activity of the oncogenic MNK/eIF4E pathway has been associated with increased synthesis of carcinogenesis-supporting proteins via activation of mRNA nuclear export $(14,15)$ or translation $(16,17)$. Furthermore, targeting MNK1 was found to reduce GBM growth in vivo (18) and, more recently, depletion of MNK1 kinase in glioma cells increased their sensitivity to the mTORC1 inhibitor rapamycin, suggesting that regulation of eIF4E via hyperactivated $\mathrm{mTORC1}$ and MNK signaling pathways is instrumental in mediating changes in protein synthesis that support gliomagenesis (19).

In the present study, we found that 4EBP1 phosphorylation at Ser65 and the association of 4EBP1 with eIF4E are regulated 
A

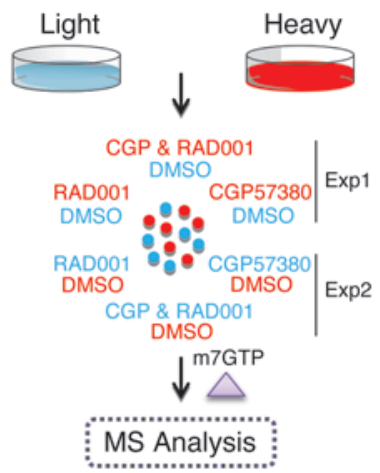

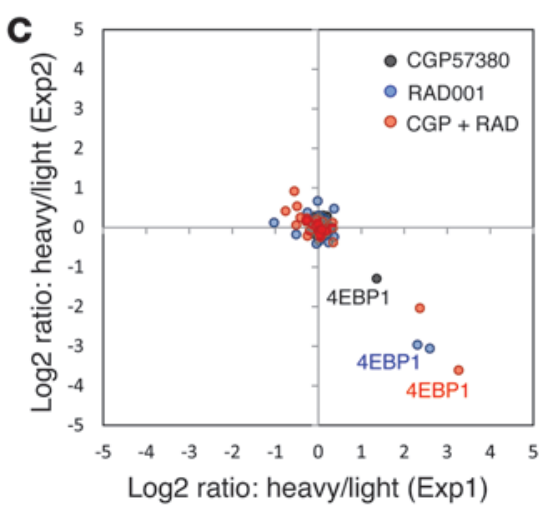

D

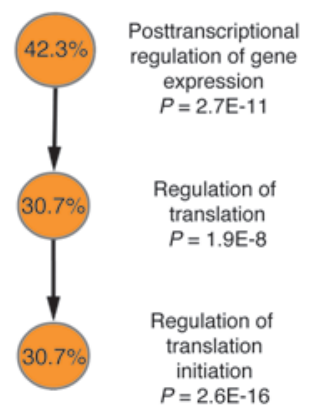

$\mathbf{E}$

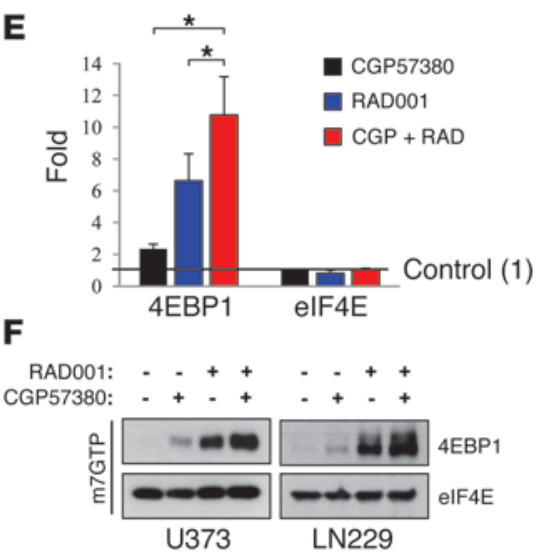

G

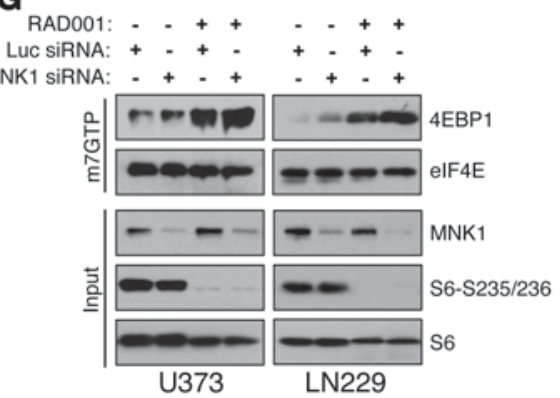

\section{Figure 1}

Targeting MNK1 increases 4EBP1 association with elF4E in RAD001-treated glioma cells. (A) Experimental design — isotopically labeled (heavy, Exp1) or unlabeled (light, Exp2) U373 cells were treated with $10 \mu \mathrm{M}$ CGP5730 and/or $10 \mathrm{nM}$ RAD001 for 20 hours, and protein lysates were combined with lysates from DMSO-treated control cells as indicated and subjected to $\mathrm{m}^{7}$ GTP-mediated precipitation of translation initiation complexes, followed by mass spectrometry (MS). (B) Phosphorylation of elF4E and S6 ribosomal protein was monitored by immunoblotting using phospho-specific antibody. Blots were stripped and reprobed with elF4E, S6, and tubulin to check for equal loading. (C) Protein abundance changes and (D) gene ontology analysis of precipitated protein complexes identified by mass spectrometry analysis. (E) Fold change of $\mathrm{m}^{7} \mathrm{GTP}$-precipitated 4EBP1 and elF4E proteins from inhibitor-treated cells compared with control cells incubated with DMSO. Data represent mean \pm SD. ${ }^{*}<0.05$. (F) Western blot analysis of $\mathrm{m}^{7}$ GTP precipitates from CGP5730- and/or RAD001-treated U373 and LN229 cells using 4EBP1- and elF4E-specific antibodies. (G) Western blot analysis for 4EBP1 and elF4E (as described above) of $\mathrm{m}^{7}$ GTP precipitates from U373 and LN229 cells that were treated for 20 hours with 10 nM RAD001 24 hours after transfection with duplex siRNA oligonucleotides against MNK1 or control duplex against luciferase. MNK1 knockdown and the inhibition of S6 phosphorylation was confirmed using MNK1-, S6-, and phospho-S6-specific antibodies on whole protein lysates (input).

via the activated MNK1 signaling pathway during the response of glioma cells to mTORC1 inhibitor RAD001. Simultaneous inhibition of mTORC1 and MNK1 efficiently inhibited protein synthesis, glioma cell proliferation, and in vivo tumor growth in GBM mouse models. Immunohistochemical analysis showed high phosphorylation of 4EBP1 at Ser65 in patients with GBM, suggesting the existence of a point of mTORC1 and MNK1 pathway convergence with therapeutic potential.

\section{Results}

Activated MNK1 influences 4EBP1 phosphorylation and association with eIF4E in response to RAD001 treatment. In a previous study, concomitant treatment of glioma cells with MNK inhibitor CGP57380 and rapamycin caused a greater decrease in polysomal levels than single compound incubation, suggesting inhibition of global protein synthesis (19). To investigate this point further, we analyzed precipitated translation initiation complexes from stable isotope labeling with amino acids in cell culture-labeled (SILAC-labeled) U373 glioma cells that were treated with CGP57380 and/or the rapamycin derivative RAD001, followed by quantitative mass spectrometry (Figure 1A). Prior to precipitation, SILAC-labeled protein lysates showed approximately $97 \%$ incorporation of heavy isotope amino acids (data not shown). The effects of inhibitor treatments were analyzed by immunoblotting in protein lysates obtained from both labeled "heavy" and unlabeled "light" cells. As shown in Figure 1B, there was a marked decline in the phosphorylation of eIF4E at Ser209 and in ribosomal protein S6 at Ser235/236 after CGP57380 and RAD001 treatments, respectively. Increased eIF4E phosphorylation was observed after inhibition of mTORC1 by RAD001 in U373 and LN229 cells, and further analysis of MNK-regulating ERK and p38 kinases showed increased phosphorylation of ERK1/2 and MNK after incubation with RAD001 in U373 cells (Figure 1B and Supplemental Figure 1; supplemental material available online with this article; doi:10.1172/ JCI70198DS1). Mass spectrometry analysis of precipitated protein complexes from CGP57380- and/or RAD001-treated cells using $\mathrm{m}^{7} \mathrm{GTP}$-sepharose identified 34 proteins (Figure 1C and Supplemental Table 1). Gene ontology analysis of these proteins showed the highest enrichment of genes involved in posttranscriptional regulation of gene expression and translation, including the ini- 


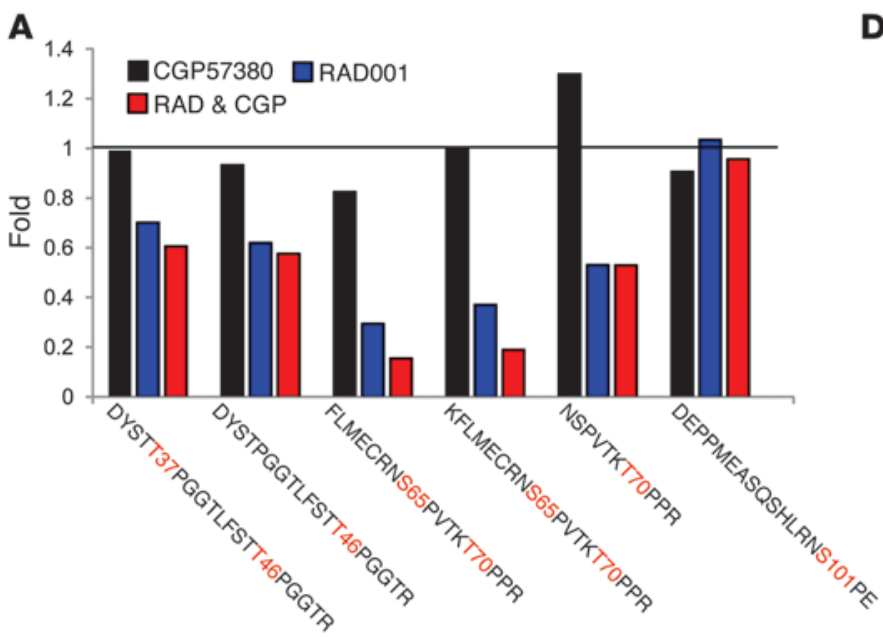

D

B

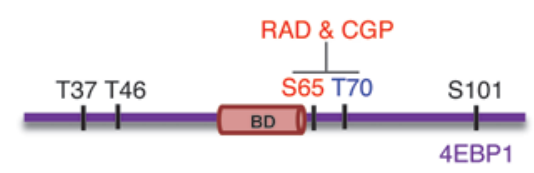

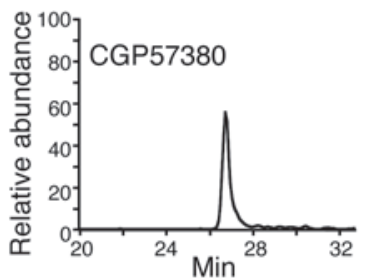

E
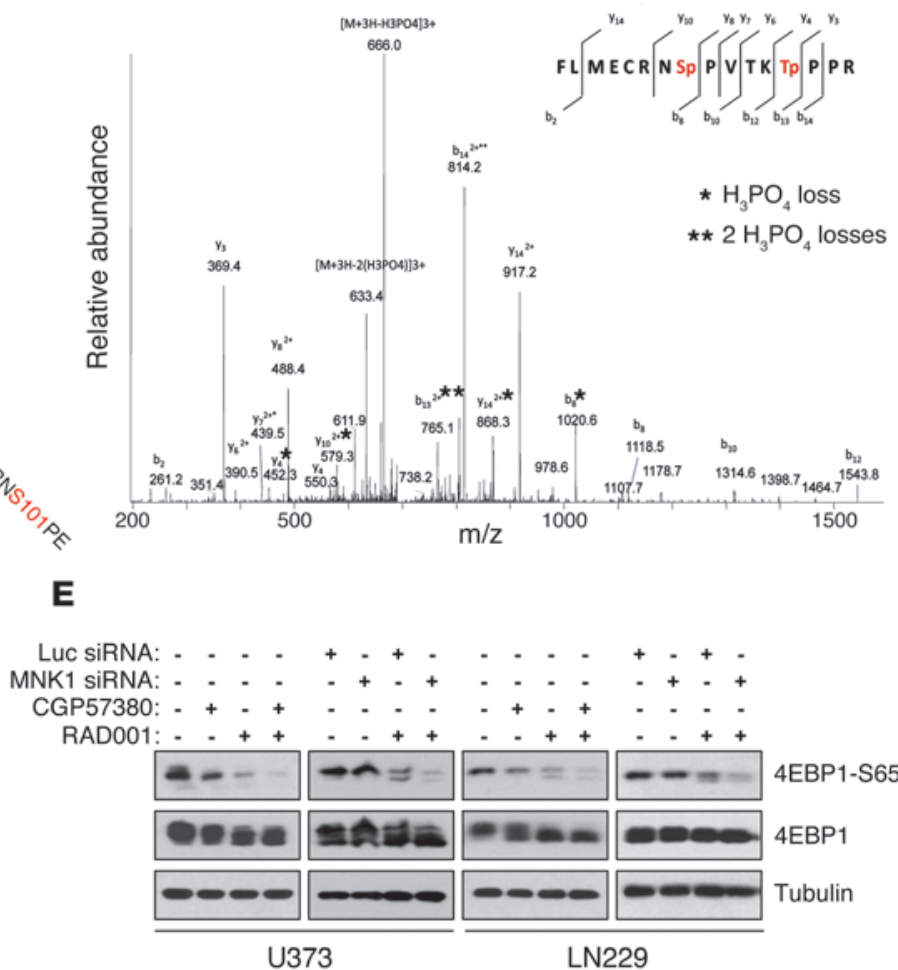

$\mathbf{F}$

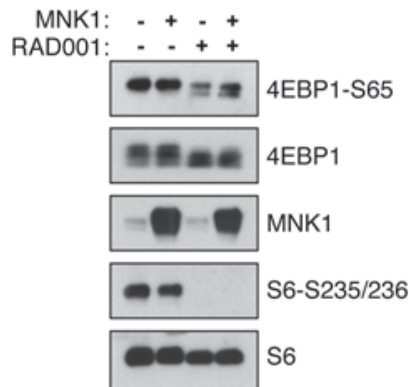

Figure 2

MNK1-dependent 4EBP1 phosphorylation at Ser65 in RAD001-treated glioma cells. (A) LN229 cells were treated with $10 \mu \mathrm{M}$ CGP5730 and/or 10 nM RAD001 for 2 hours, and 4EBP1 immunoprecipitated using a 4EBP1-specific antibody was analyzed by LC-MS/MS. Bars represent relative quantification of phosphopeptides. The abundance of each identified phosphopeptide in control DMSO-treated cells was set to 1. (B) Representation of the 4EBP1 protein showing phosphorylation sites affected by inhibitor treatment. BD, elF4G binding domain. (C) Relative abundance and (D) MS2 spectra for peptides covering 4EBP1 phosphorylation sites at Ser65/Thr70. Detected y-and b-ions are indicated in the sequence and $\mathrm{H}_{3} \mathrm{PO}_{4}$ loss is marked with asterisks. (E) Phosphorylation of 4EBP1 at Ser65 was analyzed by immunoblotting using a phospho-specific antibody in whole protein lysates prepared from U373 and LN229 cells after 20 hours of treatment. Blots were stripped and reprobed with 4EBP1 and/or tubulin-specific antibodies. Twenty-four hours after transfection with duplex siRNA oligonucleotides against MNK1 or control duplex against luciferase, cells were treated with $10 \mathrm{nM}$ RAD001 for a further 20 hours and lysates were subjected to Western blot analysis. (F) 4EBP1 phosphorylation in MNK1-overexpressing U373 cells 24 hours after transfection and treatment with 10 nM RAD001 was analyzed by immunoblotting as described above.

tiation step (Figure 1D). In agreement with previous models (6), association of eIF4E-binding proteins 4EBP1 and 4EBP2 was influenced by mTORC1 inhibition. Interestingly, 4EBP1 association was significantly increased by the MNK inhibitor (2.3-fold), with an additive effect when combined with mTORC1 inhibition (RAD001 alone, 6.6-fold change; RAD001 with CGP57380, 10.7 -fold change). There were no significant changes in precipitated levels of eIF4E or other translation initiation factors (Figure 1E and Supplemental Table 1). In these experiments, we used the previously described inhibitor of MNK kinases, CGP57380 (20). Currently available pharmacological MNK inhibitors, although efficiently inhibiting MNK activity and eIF4E phosphorylation, can also show activity toward other kinases. In vitro protein kinase assays had confirmed that CGP57380 inhibits MNK1 and 
A

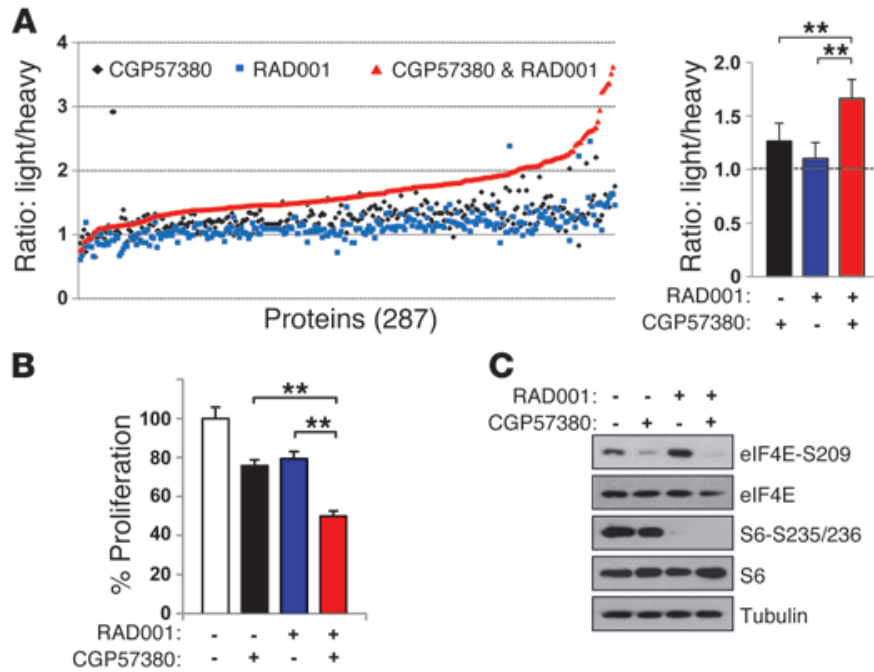

D

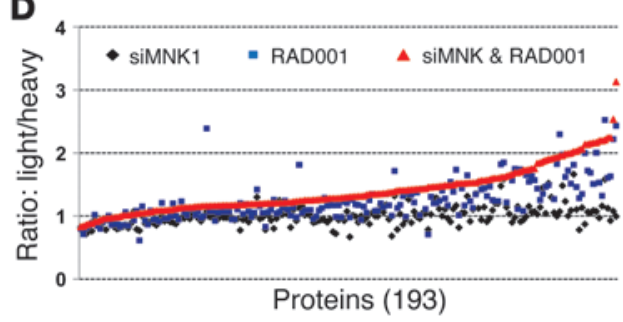

$\mathbf{E}$

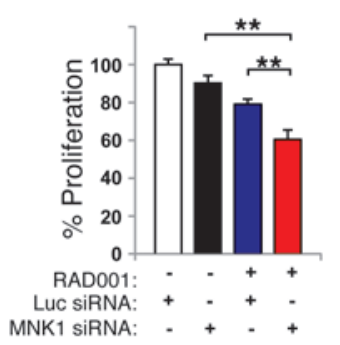

Figure 3

Concomitant targeting of $\mathrm{mTORC} 1$ and MNK1 increases inhibition of protein synthesis. (A and D) Bulk protein synthesis in LN229 cells (A) incubated with $10 \mu \mathrm{M}$ CGP5730 and/or with $10 \mathrm{nM}$ RAD001 or (D) 48 hours after transfection with siRNA against MNK1 or with a control duplex against luciferase and/or with $10 \mathrm{nM}$ RAD001, as measured by heavy lysine and arginine incorporation during an 8-hour SILAC-labeling period and mass spectrometry-based analysis. Graph points represent ratios of light- to heavy-labeled proteins for each protein identified from treated and/or transfected cells. The ratios in control (A) DMSO-treated and (D) luciferase-transfected cells were set to 1. Averages of light-to-heavy ratios \pm SD for all identified proteins are also shown. (B and $\mathbf{E}$ ) An MTTbased assay for LN229 cell proliferation (B) 3 days after treatment with $10 \mu \mathrm{M}$ CGP57380 and/or incubation with $10 \mathrm{nM}$ RAD001 or (E) after transfection with siRNA against MNK1 or luciferase control duplex and/ or incubation with $10 \mathrm{nM}$ RAD001. Results were assayed in triplicate and are shown as percentage proliferation compared with control cells. Data represent mean \pm SD. (C) Phosphorylation of S6, elF4E, and (F) MNK1 knockdown was monitored by Western blot analysis. For equal loading control blots were stripped and reprobed with elF4E-, S6-, and tubulin-specific antibodies. ${ }^{*} P<0.01$.
MNK2 with $\mathrm{IC}_{50}$ values of 0.87 and $1.6 \mu \mathrm{M}$, respectively (21). CGP57380 also inhibits CK1, BRSK2, Aurora B, Lck, SGK, and DYRK at a similar range of $\mathrm{IC}_{50}$ values $(0.5-3.2 \mu \mathrm{M})$. In order to validate the results obtained using CGP57380, we used siRNAs for depletion of MNK1 expression that target MNK1a and MNK1b splice variants encoding identical catalytic domains. Western blot analysis using 4EBP1- and eIF4E-specific antibodies showed the highest 4EBP1 levels in $\mathrm{m}^{7} \mathrm{GTP}$-precipitated complexes from CGP57380-treated (Figure 1F) or MNK1 siRNA-transfected (Figure 1G) and U373 and LN229 glioma cells with RAD001 treatment, without changes in eIF4E levels.

This additive effect clearly suggests that, in addition to mTORC1 signaling, the MNK1 pathway also affects the 4EBP1 phosphorylation that determines binding to eIF4E. To investigate this point further, we immunoprecipitated endogenous 4EBP1 proteins from control-treated or CGP5738- and/or RAD001-treated LN229 cells, and the precipitated 4EBP1s were analyzed by mass spectrometry. Unique phosphopeptides were identified (Supplemental Figure 2 and Figure 2D) and quantified. As shown in Figure 2, A-C, a 2-hour incubation with RAD001 reduced the level of peptides covering phosphorylation sites at Thr37/46 to only moderate levels of $62 \%$ to $70 \%$ of the control, whereas peptides covering phosphorylation sites at Ser65 and Thr70 were most affected by RAD001 treatment (29\%-53\% of the control). Phosphorylation at Ser101 was insensitive to any treatment. CGP5738 treatment alone showed only a minor inhibitory effect on peptides covering phosphorylation sites at Ser65 and Thr70 (82\%) compared with the substantial effect of CGP57380 treatment combined with RAD001 treatment (15\%-18\% of the control) (Figure 2, A and C). Furthermore, analysis of peptides covering only the phosphorylated Thr70 site showed no inhibition upon CGP57380 treatment alone or in combination with RAD001, suggesting that the phosphorylation site of 4EBP1 at Ser65 is affected by the MNK1 pathway as well as by mTORC1. To test this observation, we used Western blot analysis using phospho-specific antibodies. Treatment of U373 and LN229 cells with CGP57380 alone for 20 hours reduced 4EBP1 phosphorylation at Ser65 only moderately, but targeting MNK1 with CGP57380 or with siRNA against MNK1 in combination with RAD001 markedly inhibited the same phosphorylation site (Figure 2E), while overexpression of full-length MNK1 in U373 cells partially rescued inhibition of Ser65 phosphorylation of 4EBP1 in the presence of RAD001 (Figure 2F).

Since 4EBP1 phosphorylation can be entirely blocked by previously reported ATP-competitive inhibitors, such as Torin2 (22), which targets mTOR in both complexes (in contrast to rapalogs that affect mTORC1 activity), we analyzed whether RAD001/CGP57380 treatment can influence mTOR activity within mTORC2. As shown in Supplemental Figure 3, Torin2 efficiently inhibited Ser473 phosphorylation of AKT (targeted by mTORC2), whereas CGP57380 alone or combined with RAD001 did not influence this phosphorylation site. Torin2 inhibited cell proliferation more efficiently as compared with RAD001, whereas RAD001 and CGP57380 combination treatment inhibited proliferation similarly to Torin2. In addition, combination treatment of Torin2 and CGP57380 had a stronger inhibitory effect on cell proliferation as compared with that of Torin2 alone, suggesting that CGP57380/RAD001 treatment influences cell proliferation similarly to Torin2, although via a distinct mechanism. 
A

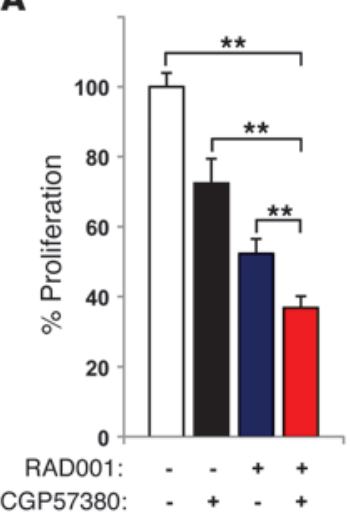

B

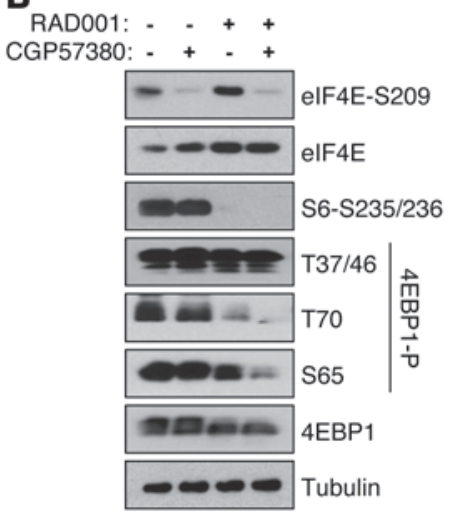

C

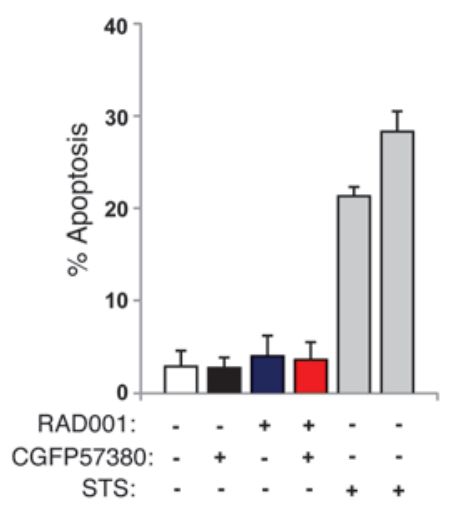

D
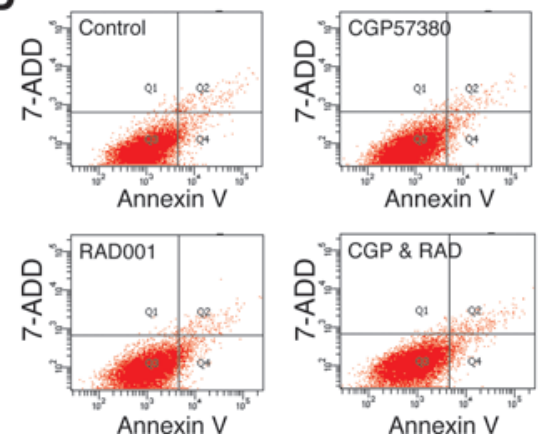

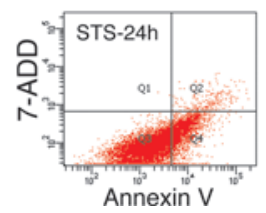

E

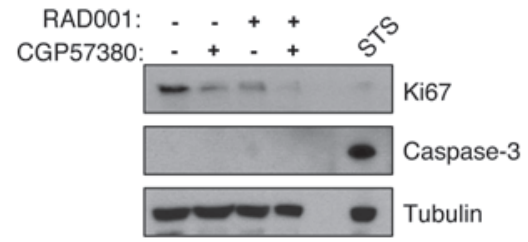

Figure 4

Inhibition of proliferation and 4EBP1 phosphorylation in RAD001- and CGP57380-treated cells. U87MG-luc cells were treated with 10 $\mu$ M CGP57380 and/or with $10 \mathrm{nM}$ RAD001 for 3 days. (A) MTT-based proliferation assays were performed in triplicate, and the results are shown as percentage proliferation compared with control DMSO-treated cells. Data represent mean $\pm \mathrm{SD}$. ${ }^{*} P<0.01$. (B) Phosphorylation of $4 \mathrm{EBP} 1$, elF4E, and S6 protein was analyzed by immunoblotting using phospho-specific antibodies. For equal loading, blots were probed with antibodies against total elF4E, 4EBP1, and tubulin. (C) Apoptosis was assessed in triplicate by annexin V and 7-ADD staining followed by flow cytometry, and percentage apoptosis is shown as percentage of annexin V-positive cells. Data represent mean $\pm \mathrm{SD}$. Cells incubated with $1 \mu \mathrm{M}$ staurosporine (STS) for 16 and 24 hours were used as a positive control. (D) Dot plots are displayed with annexin V and 7-ADD staining. Lower- and upper-right quadrants represent early and late apoptotic cells, respectively. (E) Western blot analysis for Ki67 and cleaved caspase-3 in treated cells, as described above. Tubulin was used as a loading control.

Targeting MNK1 inbibits protein synthesis and proliferation in RAD001treated cells. To investigate further whether MNK1-dependent regulation of 4EBP1 influences global protein synthesis in RAD001treated cells, we monitored the incorporation of heavy-labeled amino acids into newly synthesized proteins using the SILAC technique and mass spectrometry. As shown in Figure 3A and Supplemental Figure 4, 2- or 8-hour treatment of LN229 or U373 cells with CGP57380 and RAD001 had a much greater inhibitory effect on global protein synthesis than single compound treatments. Similarly, RAD001 treatment of LN229 cells depleted of MNK1 by siRNA had a much greater inhibitory effect than treatment of control-transfected cells with RAD001 alone (Figure 3D). Furthermore, treatment with either CGP57380 or RAD001 reduced glioma cell proliferation, and their combination had an additive effect (Figure 3, B and E). Western blotting confirmed inhibition of the phosphorylation of ribosomal protein $\mathrm{S} 6$ and eIF4E as well as efficient MNK1 knockdown (Figure 3, C and F).

In addition, as our data (Supplemental Figure 1) indicated that RAD001 increases ERK activity, we also studied inhibition of ERK and mTORC1 pathways in glioma cells. Similarly to that after CGP57380 and RAD001 treatment, we observed an additive inhibitory effect on proliferation in glioma cells incubated with RAD001 and MEK inhibitor U0126 (Supplemental Figure 5). Furthermore, to analyze whether the MNK1-dependent resistance to rapalogs is mediated by its effect on 4EBP1 or eIF4E, we used a previously reported (23) tetracycline-inducible expression construct, which constitutively binds to eIF4E, for a 4EBP1 mutant in which 4 phosphorylation sites were replaced with alanine (T37A, T46A, S65A, and T70A). As shown in Supplemental Figure 6, tetracycline-induced expression of this 4EBP1 mutant inhibited U373 cell proliferation, whereas overexpression of the eIF4E S209A mutant or MNK inhibitor CGP57380 did not further increase the inhibitory effect of the 4EBP1 mutant, suggesting that phosphorylation of 4EBP1 plays a major role in the regulation of cell proliferation.

Combined treatment with CGP57380 and RAD001 additively inhibits tumor growth in an orthotopic GBM mouse model. To evaluate the effects of CGP57380 alone or in combination with RAD001 in vivo, we took advantage of a previously established U87MG-luc glioma cell line stably expressing firefly luciferase (24). In our ex vivo preliminary analysis, concomitant treatment with CGP57380 
A

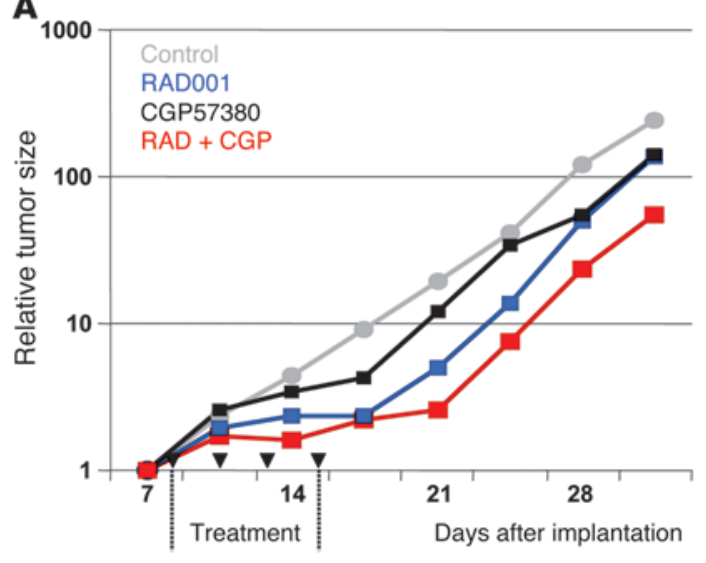

B

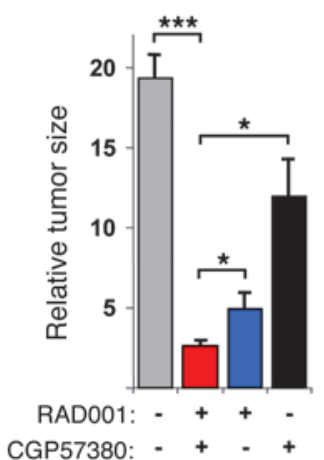

Day 21
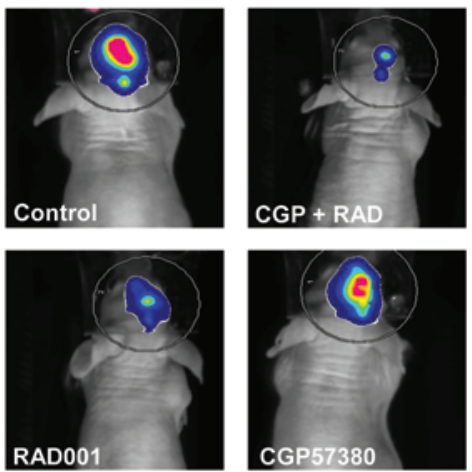

C

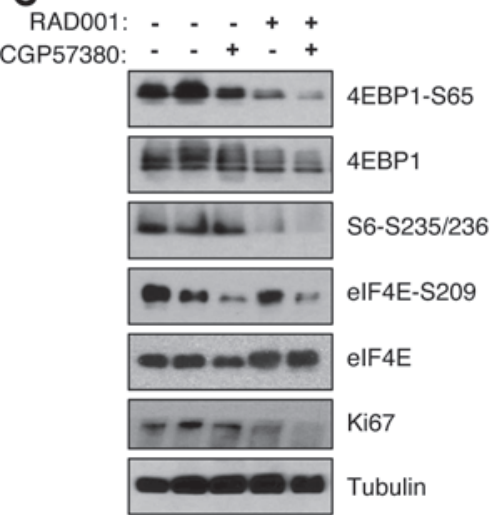

D

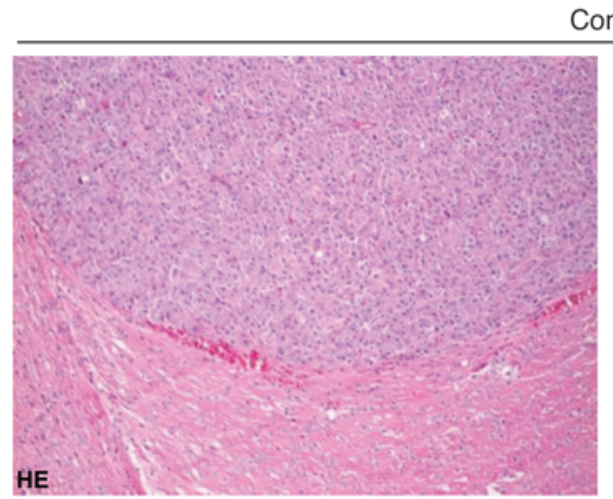

Control

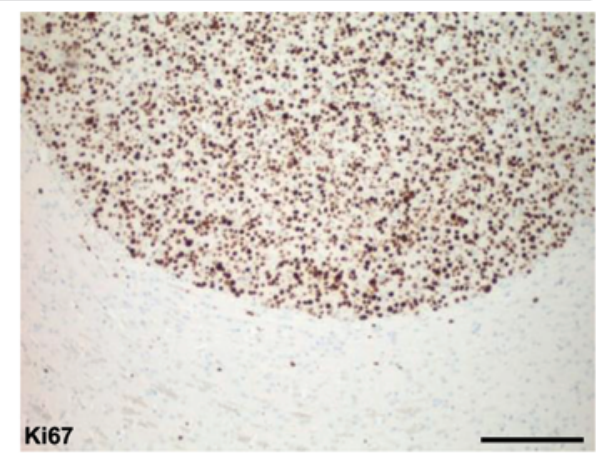

Ki67

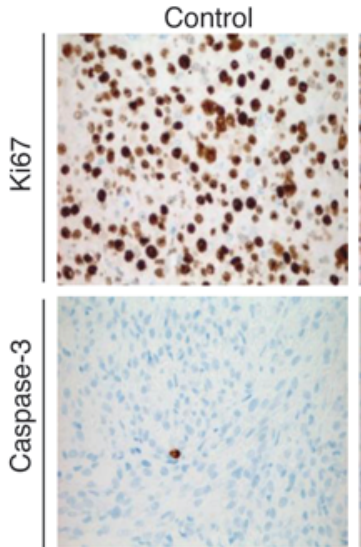

CGP57380
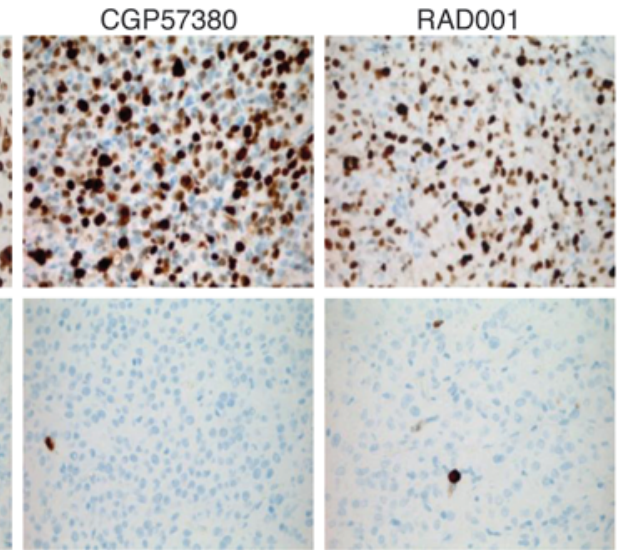

$\mathrm{CGP}+\mathrm{RAD}$
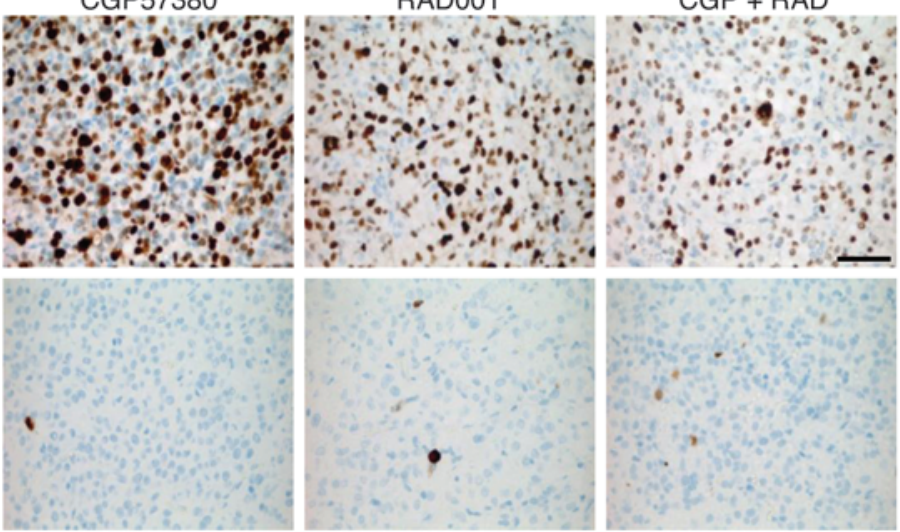

\section{Figure 5}

Concomitant treatment with CGP57380 and RAD001 inhibits tumor growth in vivo. (A) Growth curves for an orthotopic GBM xenograft nude mouse model. U87MG-luc glioma cells were implanted into the brains of immunocompromised (nude) mice. Compound-treated and control animal groups $(n=5)$ received 4 injections of CGP57380 or/and RAD001 between days 8 and 15 after implantation as indicated by black triangles, and tumor growth was monitored and analyzed by noninvasive BLI over a period of 31 days. (B) Relative tumor size 21 days after implantation and representative BLI images for treated and control animals. Data represent mean \pm SD. (C) Phosphorylation of 4EBP1, elF4E, and S6 protein and Ki67 expression analyzed by immunoblotting in brain tumors from control and treated mice dissected 1 day after the final injection, as described above. (D) H\&E staining and IHC for Ki67 and cleaved caspase-3 in control and treated brain tumors. Scale bars $50 \mu \mathrm{m}$ (top); $200 \mu \mathrm{m}$ (bottom). (E) The percentage of Ki67-positive cells was determined by counting stained cells in the treated tumors. Bars represent mean \pm SD. ${ }^{\star} P<0.05$, ${ }^{\star *} P<0.01,{ }^{* * *} P<0.001$. 
and RAD001 had a markedly greater effect on the inhibition of U87MG-luc cell proliferation (Figure 4A), associated with the most effective inhibition of 4EBP1 phosphorylation at Ser65, than single drug treatments (Figure 4B).

FACS analysis of Annexin $\mathrm{V}$ - and 7-ADD-stained cells after treatment did not indicate significant changes in apoptosis levels (Figure 4, C and D). Further, Western blot analysis showed the lowest level of proliferation marker Ki67 in RAD001- and CGP57380treated cells, while cleaved caspase-3 expression was not detected (Figure 4E). For in vivo analysis, cells were implanted into the brains of immunocompromised nude mice, and tumor growth was monitored by noninvasive bioluminescence imaging (BLI). Treated animals received 4 injections of inhibitors between days 8 and 15 after implantation. The relative tumor growth curves for control-, CGP57380-, and/or RAD001-treated animals are shown in Figure 5A. Control tumors showed exponential growth, and mice with these tumors had a median survival of 28 days. Treatment with CGP57380 alone significantly reduced tumor growth $(P=0.017)$ as did RAD001 alone $(P=0.002)$. In both cases, tumor growth inhibition was observed from day 10 to 17 ; exponential tumor growth resumed later as a result of the cession of drug administration on day 15. The CGP57380 and RAD001 combination treatment led to the most pronounced growth-inhibitory effect $(P=0.009)$, which lasted until day 21 (Figure 5, A and B). As shown in Table 1, the effect of the combined treatment was significantly greater than that of the individual drugs $(P<0.05)$, indicating that CGP57380 together with RAD001 had the most effective antiglioma activity in this in vivo GBM model. Western blot analysis using phospho-specific antibodies showed reduced eIF4E or S6 phosphorylation after CGP57380 or RAD001 treatments, respectively, whereas combined drug treatment resulted in the most pronounced inhibition of 4EBP1 phosphorylation at Ser65 as well as Ki67 expression in whole protein lysates prepared from treated tumors (Figure 5C). Together with immunohistochemistry (IHC), H\&E staining for Ki67 on tissue sections marked tumor areas clearly, and the lowest number of Ki67-positive cells was observed in tumors treated with a combination of CGP57380 and RAD001 (Figure 5, D and E). Immunostaining for cleaved caspase-3 revealed very low counts of apoptotic cells $(<2 \%)$ within the tumor masses, suggesting a cytostatic effect of the treatment (Figure 5D).

Increased 4EBP1 phosphorylation at Ser65 in GBMs. As reported previously, the activated mTORC1 and MNK1 pathways support gliomagenesis, and our present results suggest that these 2 pathways converge at and regulate 4EBP1. To investigate this point in patients with glioma, we analyzed 4EBP1 phosphorylation at Ser65 as well as total 4EBP1 protein levels in 58 patients with GBM as well as normal brain samples. Both total and phosphorylated 4EBP1 showed moderate to high expression levels in the vast majority of tumors. Staining for total 4EBP1 showed strong cytoplasmic and weak nuclear staining in 57 of 58 (98\%) gliomas, whereas phospho-4EBP1 was detected predominantly in the nuclei of 54 of 58 (93\%) tumors (Figure 6, A, B, and E-G), with the most intense staining observed in mitotic figures (Figure 6, $\mathrm{G}$ and $\mathrm{H}$ ). In addition, an increase in immunostaining in mitotic figures was also observed for phospho-MNK1 in GBM tissue sections (Supplemental Figure 7), further suggesting that activated MNK1 increases 4EBP1 phosphorylation at Ser65. Strong total 4EBP1 and phospho-4EBP1 immunostaining was scored in corresponding tumor areas on consecutive tissue sections of solid tumors (Supplemental Figure 7 and Figure 6, I and J), whereas

\section{Table 1}

$P$ values for growth curves shown in Figure 5A comparing all experimental conditions

$\begin{array}{lc}\text { Treatment } & \boldsymbol{P} \text { value } \\ \text { Control vs. RAD and CGP } & 0.009 \\ \text { Control vs. RAD001 } & 0.002 \\ \text { Control vs. CGP57380 } & 0.017 \\ \text { RAD and CGP vs. RAD001 } & 0.042 \\ \text { RAD and CGP vs. CGP57380 } & 0.016 \\ \text { RAD001 vs. CGP57380 } & 0.039\end{array}$

CGP, CGP57380; RAD, RAD001.

surrounding (nonsolid) tumor areas, such as infiltrating zone, peritumoral stroma and parenchymal cells (Figure 6, A, B and G), or normal brain tissue (Figure 6, C and D), showed a lower level of phosphorylated 4EBP1. There was no significant correlation between total 4EBP1 and phospho-4EBP1 levels in the analyzed GBM samples, suggesting that hyperactivated mTORC1 and/or MNK1 pathways in human gliomas increase 4EBP1 phosphorylation without affecting 4EBP1 protein level.

To investigate this point further, we analyzed expression levels of phospho-4EBP1 (Ser65), together with phospho-S6 (a marker of mTORC1 activity) and phospho-eIF4E (a marker for MNK activity), by IHC in selected corresponding tumor areas of 16 primary GBMs. All tumor sections were subjected to immunostaining for expression of 2 well-known glioma-relevant tumor suppressors (p53 and PTEN) as well as for the prognostic marker IDH1 R132H mutant (25). As show in Figure 7, staining patterns for phospho-4EBP1 were similar to those of phospho-S6, which presumably reflects high activity of mTORC1 in human gliomas. High levels of phospho-4EBP1 as well as of phospho-eIF4E were detected within identical tumor areas, and, additionally, phospho-eIF4E was also found in other phospho-4EBP $1_{\text {low }}$ tumor regions. We have not observed an association between phospho-4EBP1 and p53 or PTEN staining. Furthermore, all analyzed tumor sections were negative for the IDH1 mutant, as expected for primary GBMs.

\section{Discussion}

During gliomagenesis, genetic alterations promote brain tumor development and disease progression via hyperactivation of receptor tyrosine kinases, including EGFR, PDGFRA, ERBB2, and MET receptors. This leads to the activation of 2 major oncogenic pathways, PI3K/AKT/mTOR and RAS/RAF/MEK/ERK. In addition, $P T E N$ inactivation by mutation or homozygous deletions as well as PI3K-activating mutations are found in $36 \%$ and $15 \%$ of human gliomas, respectively, further increasing signaling via tumorigenic pathways (1). Previous studies in various human cancer models, including gliomas, reported that individual targeting of the PI3K/ $\mathrm{AKT} / \mathrm{mTOR}$ or RAS/ERK/MEK pathways was not sufficient to inhibit tumor growth and that depletion of one pathway produced tumors promoted by the other pathway $(26,27)$. This suggests the existence of molecular convergence points that potentially influence a common set of substrates to support carcinogenesis and survival. For example, the prosurvival effects of the activated PI3K/ $\mathrm{AKT}$ and ERK/MEK pathways integrate at the BAD protein, the phosphorylation of which by both pathways prevents its apoptotic activity by binding to chaperone protein 14-3-3 and sequestering 

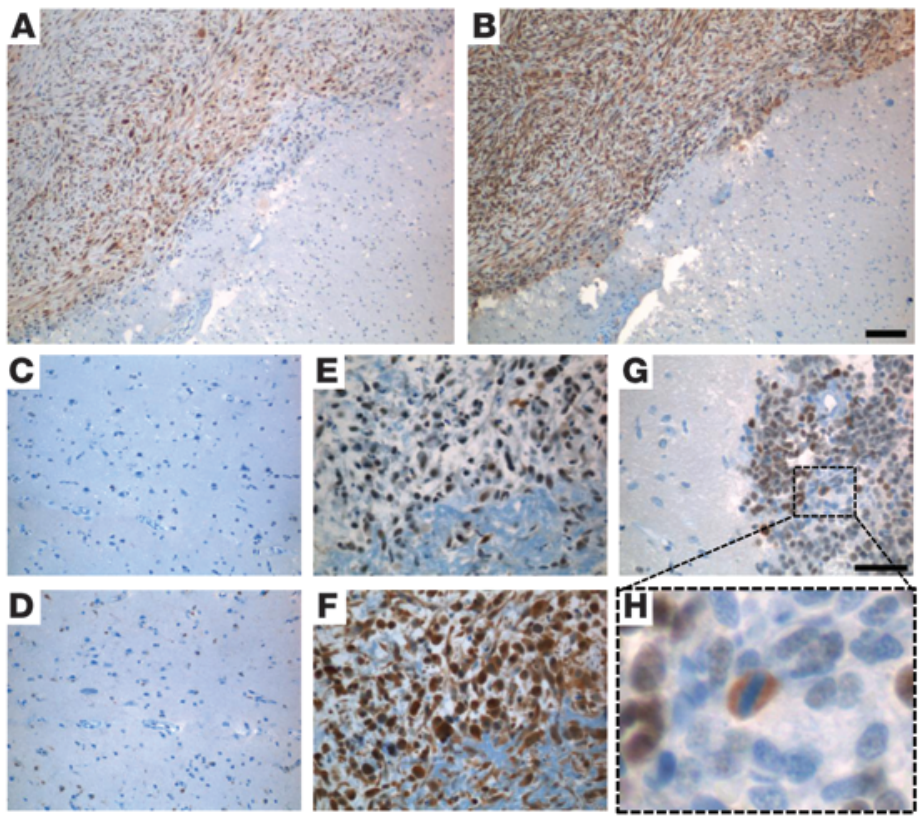

I

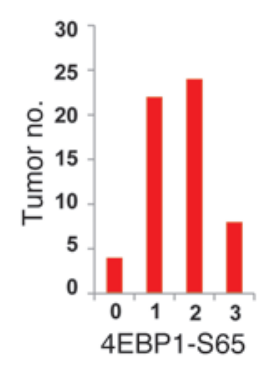

J

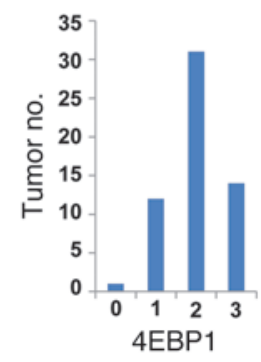

Figure 6

Increased 4EBP1 phosphorylation at Ser65 in GBM samples. Immunostaining (A, C, E, G, and H) for phosphorylated 4EBP1 at Ser65 and (B, D, and F) for total 4EBP1. (C and D) GBM samples and normal control brain samples were stained with specific monoclonal antibodies (brown) and counterstained with hematoxylin (blue). (H) Higher-magnification view of the boxed region in $\mathbf{G}$, showing an increased signal for phospho-4EBP1 in mitotic cells (original magnification, $\times 400)$. Scale bar: $50 \mu \mathrm{m}(\mathbf{A}$ and $\mathbf{B})$; $100 \mu \mathrm{m}(\mathbf{C}-\mathbf{G})$. Immunohistological scoring of (I) phospho-4EBP1 and ( $\mathbf{J})$ total 4EBP1 in 58 primary GBM samples. phosphorylated BAD in the cytoplasm (28). Thus, efficient therapy in this case requires the simultaneous inhibition of both PI3K/ AKT and MAPK pathways to impair their downstream oncogenic effects. MNK kinases are activated by ERKs and p38, whereas the $\mathrm{PI} 3 \mathrm{~K} / \mathrm{AKT}$ pathway regulates mTORC1 activity. This suggests that inhibition of MNKs and mTORC1 could reproduce the effects of $\mathrm{MEK} / \mathrm{ERK}$ and PI3K/AKT inactivation in cancers. Indeed, combined pharmacological targeting of MTORC1 and MNKs by rapalogs and CGP57380, respectively, shows greater growth inhibitory effects than the suppression of one of the pathways in lung and prostate cancer cells and, more recently, in glioma and lymphoma cells $(16,19,29,30)$. In the latter studies, analysis of polysomal profiles from MNK inhibitor- and rapamycin-treated prostate cancer and glioma cells revealed a significant drop in polysome levels, indicating that the simultaneous inhibition of the mTORC1 and MNK1 pathways strongly affects global protein synthesis $(16,19)$. In support of these observations, we found MNK1-dependent phosphorylation levels of translation regulator 4EBP1, as well as its association with eIF4E, during the response of glioma cells to mTORC1 inhibition by RAD001. Similarly, recent studies in human breast cancer and colorectal cells with PIK3CA and KRAS mutations identified 4EBP1 as a key integrator of the activated PI3K/AKT and ERK pathways (31). Concomitant treatment with AKT and MEK inhibitors (AKTi and PD0325901, respectively) increased cell cycle arrest and apoptosis and was required for the effective inhibition of cap-dependent translation and protein synthesis associated with 4EBP1 dephosphorylation. In the same study, overexpression of an active 4EBP1 mutant with 4 phosphorylation sites replaced by alanine (Thr36/46/70 and Ser65), which constitutively binds eIF4E, suppressed colorectal tumor growth in vivo. This indicates that 4EBP1 inactivation by phosphorylation triggers an oncogenic effect via eIF4E and the regulation of protein synthesis. Consistent with the previous results, we found marked decrease in protein synthesis in glioma cells associated with the most efficient 4EBP1 dephosphorylation only when either mTORC1 or MNK1, regulated by the MEK/ERK pathway, were inhibited by RAD001 and CGP57380 or by MNK1-specific depletion. Furthermore, overexpression of an eIF4E-phospho mutant or addition of the MNK inhibitor CGP57380 did not significantly increase the inhibitory effect of the 4EBP1-phospho mutant, suggesting that phosphorylation of 4EBP1 plays a major role in glioma cell proliferation via translation regulation. Finally, our study in an orthotopic in vivo GBM mouse model confirmed the inhibitory effect of concomitant treatment with RAD001 and CGP57380 on tumor growth, which is associated with a low level of 4EBP1 phosphorylation. Thus, our present data and the former study suggest that the oncogenic activities of the PI3K/AKT/ mTOR and RAS/ERK/MNK pathways converge at 4EBP1 and influence global protein synthesis during gliomagenesis.

Increased 4EBP1 phosphorylation was previously reported in breast, ovarian, and prostate cancers as well as in melanomas, in which its activity correlated with malignant progression and poor prognosis (32-34). Our immunohistochemical analysis showed high levels of 4EBP1 phosphorylation at Ser65 in human GBM samples and an association between S6 and 4EBP1 phosphorylation levels (both regulated by mTORC1), presumably reflecting high mTORC1 activity in malignant gliomas. We observed especially high levels of phospho-4EBP1 at Ser65 in mitotic figures, consistent with a previous study reporting increased 4EBP1 phosphorylation during mitosis on Ser65 and Thr70, with complete dissociation from eIF4E (35). This was suggested as a mechanism by which immediate protein synthesis is initiated in HeLa cells in the early $G_{1}$ phase of the cell cycle. Notably, we also observed high levels of phosphorylated MNK1 in mitotic figures in the same GBM sections, suggesting that MNK1 activity regulates 4EBP1 phosphorylation at Ser65 in dividing cells. Similarly, increased staining for phospho-MNK1 in mitotic HeLa cells has been noted in a very recent report, in which MNK1 activity was required for abscission and proper completion of cytokinesis (36). Nevertheless, the role of MNK1 kinase activity, with regard to 4EBP1 regulation during mitosis, as well as its consequences for glioma cell division and growth in the early $\mathrm{G}_{1}$ phase await further study. 
p53

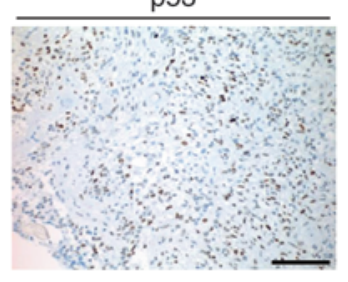

PTEN

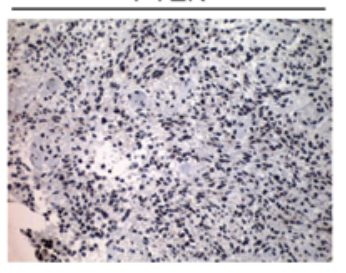

IDH1mut

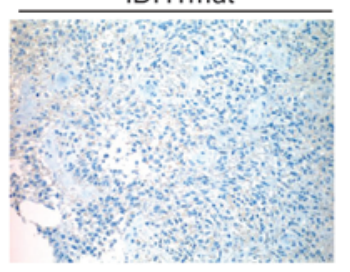

BP1-S65

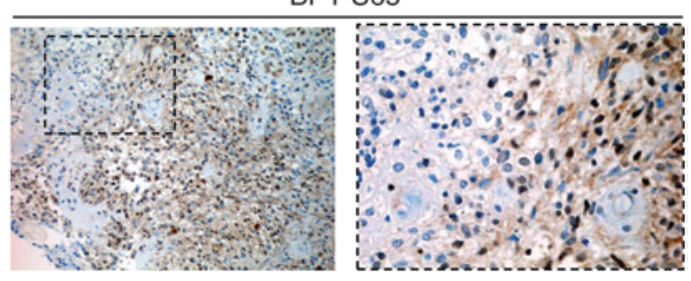

S6-S235/236

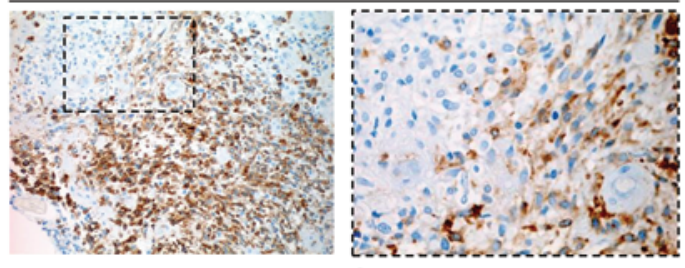

elF4E-S209

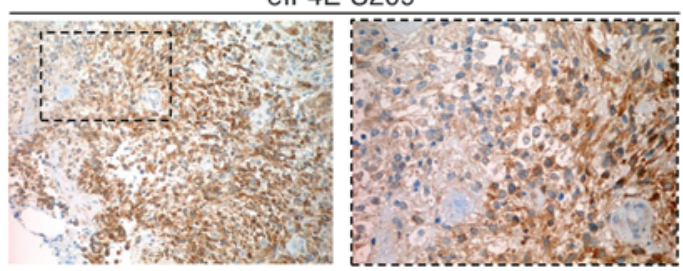

\section{Figure 7}

Phosphorylation of 4EBP1, S6, and elF4E in GBM samples. Immunostaining for p53, PTEN, and IDH1 R132H mutant as well as for phosphorylated 4EBP1, ribosomal protein S6, and elF4E was accomplished using specific monoclonal antibodies (brown) in the corresponding tumor areas. Hematoxylin (blue) was used for counterstaining. Higher-magnification views of the boxed regions in the center column are shown in the right column (original magnification, $\times 200$ ). Scale bar: $100 \mu \mathrm{m}$ (left and center columns).
A model of hierarchical phosphorylation events necessary for the dissociation of 4EBP1 from eIF4E was previously proposed, according to which phosphorylated Thr $37 / 46$ were priming sites for the subsequent phosphorylation of Thr70 and Ser65 $(37,38)$. Phosphorylation at Ser101 was also required for Ser65 modification (39). Similar to our observations, these studies, together with subsequent reports, showed that 4EBP1 phosphorylation at Thr37/46 is insensitive to rapamycin treatment, whereas modifications at Ser65 and Thr70 are strongly affected by rapalogs. Furthermore, in contrast to that at Thr37/46 sites, 4EBP1 interaction with eIF4E is directly influenced by phosphorylation at Ser65 and Thr70, which are localized close to the eIF4E-binding domain (40). Thus, lack of Ser65 and Thr70 phosphorylation prevents $4 \mathrm{EBP} 1$ dissociation and eIF4E release, which is required subsequently for eIF4F formation and cap-dependent translation initiation. Our quantitative mass spectrometry data and validation analysis showed that 4EBP1 phosphorylation at Ser65 and Thr70 was most affected as a result of mTORC1 inactivation, whereas Ser65 phosphorylation was additionally inhibited by CGP57380or MNK1-specific knockdown in RAD001-treated cells. As a consequence of the lowest level of 4EBP1 phosphorylation resulting from concomitant inhibition of the MTORC1 and MNK1 pathways, we observed the greatest increase in eIF4E/4EBP1 interaction and the most efficient inhibition of global protein synthesis.

Although mTORC1 inhibition had a major effect on 4EBP1 phosphorylation at Ser65 and Thr70 compared with MNK1 inactivation, we observed that a low level of 4EBP1 phosphorylation at Ser65 was dependent on the MNK1 pathway in RAD001-treated cells. This suggests that a lack of mTORC1 signaling activates MNK-dependent compensatory mechanisms that maintain a level of protein synthesis sufficient for cancer cell survival. In fact, we observed increased phosphorylation of MNK substrate eIF4E at Ser209 in RAD001-treated cells, indicating that inhibition of mTORC1 increases MNK activity. In agreement with our data, a previous study reported a PI3K-dependent elevation of eIF4E phosphorylation by MNKs in rapamycin- or RAD001-treated lung, colon, and breast cancer cells as well as in GBM and myeloma cells (29). This suggests that PI3K downstream signaling can activate the MNK/eIF4E pathway. In an early study (41), MNK kinases were first identified as ERK and p38 kinase interaction partners. ERK and p38 kinase phosphorylated MNKs and stimulated their activity toward the eIF4E substrate. Furthermore, stress-induced MNK1 activity was inhibited by the MEK inhibitor PD098059 or the p38 MAPK inhibitor SB202190, indicating that activated ERKs and p38 kinases regulate the MNK/eIF4E pathway (42). More recently, increased PI3K-dependent ERK activity was reported in rapamycin-treated breast and prostate cancer cells, as was elevated ERK phosphorylation in human breast cancer specimens after treatment with RAD001 (43). Consistently, our analysis of MNK-regulating kinases indicated that rapalog-activated ERKs represent a major MNK-activating pathway in RAD001-treated gliomas. Furthermore, in our study, pharmacological depletion of ERK activity had an additive effect with RAD001 on inhibition of glioma cell proliferation, similar to MNK inhibition. Thus, the observed increase in eIF4E phosphorylation by MNKs in RAD001treated cells presumably results from activation of upstream PI3K/ MAPK pathways that trigger MNK-dependent survival mechanisms. Deletion of the Mnk1 and Mnk2 genes had no significant effect on normal growth and development in a double-knockout mouse model (44), indicating that the MNK/eIF4E pathway is not required for global protein synthesis but might be advantageous under certain conditions, such as stress or cancer. Indeed, MNK1 activation may be induced by different types of stress, including radiation, whereas MNK2 shows a much higher basal activity than MNK1 (11). Thus, the activation of MNK1 may represent a survival promoting mechanism that is hyperactivated in cancers, particularly during response to cancer treatments, such as rapalogs. Supporting evidence for a MNK1 signaling activity that reduces 


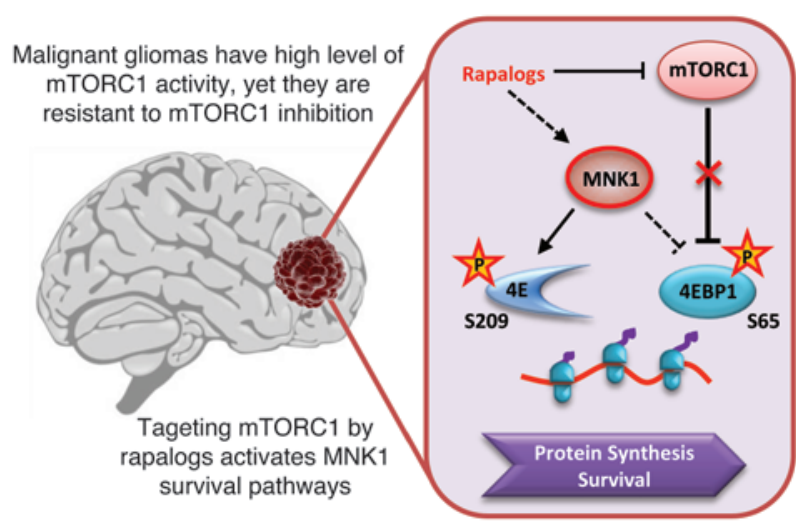

cancer cell sensitivity to certain therapies stems from a very recent report, showing that trastuzumab-resistant breast cancer cells express elevated MNK1 levels that are crucial to their survival (45).

Although MNK1 and MNK2 kinases share eIF4E as a substrate, they are apparently part of different pathways: re-introduction of wild-type Mnk1 but not Mnk2 repaired the starvation-induced apoptotic phenotype of embryonic fibroblasts from $M n k 1 / 2$ double-knockout mice (46). Contrary to MNK1 kinase, MNK2 has been shown recently to inhibit eIF4G activation and, thus, protein synthesis, suggesting that MNK2 antagonizes mTORC1 signaling and suppresses protein synthesis in skeletal muscles (47). In addition to MNK1-specific knockdown, we used CGP57380, which inhibits both MNKs in GBM cells, which normally have high mTORC1 activity. Thus, the inhibition of mTORC1-antigonizing MNK2 activity presumably had only a minor effect in the cells analyzed. In addition, MNK2 signaling and its phenotypical consequences may be different in skeletal muscle and in the transformed cells. Results of a recent study of pancreatic ductal adenocarcinoma cells treated with the chemotherapeutic agent gemcitabine suggest a prosurvival activity for MNK2 (48). Therefore, further dissection of MNK2 signaling pathways in the context of carcinogenesis is warranted.

The molecular mechanism behind MNK regulation of translation and promotion of cancer cell survival is not fully understood. Several previous studies have proposed that activated MNK1 downstream pathways increase the translation of specific mRNAs involved in carcinogenesis and survival, such as MCL1 (12); CCL2, CCL7, MMP3, and MMP9 (17); and SMAD2 (19). More recently, MNK1 activity was found to increase IRES-dependent translation of c-Myc in rapamycin-treated multiple myeloma cells, suggesting that MNK1 pathways can regulate cap-independent mechanisms of translation (49). However, not all MNK1-regulated mRNAs isolated so far have IRES elements. Therefore, translation regulation via MNK signaling might depend on specific factors, such as cancer type or stress triggered by particular therapies. Our quantitative proteomics analysis showed that inhibition of the mTORC1 pathway alone did not affect protein synthesis and that efficient inhibition of protein synthesis in RAD001-treated cells occurred only after the additional targeting of MNK1. Similarly, a recent SILAC-based analysis of rapamycin-treated cells demonstrated that global protein synthesis had little effect on general protein stability (50), suggesting that some other mTORC1-independent mechanism contributes to the preservation of translation. We hypothesize that, in addition to increasing translation of

\section{Figure 8}

Model of MNK1-mediated resistance to rapalogs. Rapalogs abrogate mTORC1-mediated inhibition of 4EBP1 phosphorylation at Ser65 and Thr70. Activated by rapalogs, the MNK1 signaling pathway phosphorylates elF4E and maintains 4EBP1 phosphorylation at Ser65. Activation of the MNK1/elF4E pathway increases translation of cancer-promoting and antiapoptotic proteins, whereas 4EBP1 phosphorylation followed by elF4E dissociation allows continued protein synthesis and, thus, cancer cell survival.

cancer-related and survival-promoting mRNAs, in the absence of mTORC1 signaling, activated MNK1 pathways sustain a level of protein synthesis via 4EBP1 regulation that enhances cancer cell survival (Figure 8). Thus, our data explain the synergistic inhibitory effects on translation and proliferation in mTORC1-inhibited and MNK1-depleted GBM cells and further suggest targeting the MNK1 pathway to sensitize malignant gliomas to rapalogs.

\section{Methods}

Cell culture, isotopic labeling, transfection, and treatments. Human glioma U373, LN229, and U87MG cells were cultured in DMEM supplemented with 10\% FCS and antibiotics at $37^{\circ} \mathrm{C}$ and $5 \% \mathrm{CO}_{2}$. SILAC was accomplished using the Pierce SILAC Protein Quantification Kit (Thermo Scientific). Briefly, cells were grown for 6 passages in DMEM medium containing 10\% dialyzed FBS in which naturally occurring "light" lysine and arginine were replaced by their isotopically "heavy" counterparts, ${ }^{13} \mathrm{C}_{6} \mathrm{~L}$-lysine $(100 \mathrm{mg} / \mathrm{l})$ and ${ }^{13} \mathrm{C}_{6}{ }^{15} \mathrm{~N}_{4} \mathrm{~L}$-arginine $(50 \mathrm{mg} / \mathrm{l})$. The medium was supplemented with L-proline at $100 \mathrm{mg} / \mathrm{l}$ to avoid metabolic conversion of heavy arginine to heavy proline. For the analysis of global protein synthesis, inhibitor-treated and/or transfected cells were SILAC labeled for 3 or 8 hours. Whole protein lysates were then prepared and subjected to mass spectrometry analysis as described below. Transfection was accomplished using Lipofectamine (Invitrogen) according to the supplier's instructions. For overexpression of full-length HA-MNK1 and 4EBP1 mutant, we used previously described constructs pcDNA3-HA-MNK1 (19) and pCW57.1-4EBP1_4xAla (Addgene plasmid 38240) (23), respectively. For eIF4E mutant overexpression, we used a previously reported (51) pBabe puro-eIF4E wild-type plasmid (Addgene plasmid 33252), in which we substituted Ser209 with alanine by using forward (FwS209A: 5'-TAAGAGCGGCGCCACCACTAAAA) and reverse (RevS209A: 5'-TTTTAGTGGTGGCGCCGCTCTTA) primers. A cell line stably expressing the 4EBP1 mutant in a tetracycline-inducible manner was established by selection on Puromycin (Invivogen) at $1 \mu \mathrm{g} / \mathrm{ml}$. For MNK1-specific knockdown, duplex siRNA against MNK1 or control duplex against luciferase (Qiagen) were used at a final concentration of $100 \mathrm{nM}$ in Optimem (Gibco): MNK1 sense (RNA 5'-AAACCUAUAGAGAUGGGCAdTdT-3') and control sense (RNA 5'-CGUACGCGGAAUACUUCGAdTdT- $3^{\prime}$ ). Cells at $40 \%$ to $60 \%$ confluency were transfected and/ or treated with an appropriate inhibitor (CGP57380 from Sigma-Aldrich, RAD001 from Novartis Pharma, Torin2 from Selleckchem, U0126 from Tocris, Staurosporine from Adipogen) dissolved in DMSO. DMSO alone was the control treatment. Cells were collected at the indicated time points and subjected to analysis and assay, as described below.

Western blotting. Antibodies against full-length MNK1 (C4C1), p-MNK1 (T197/202), eIF4E (C46H6), p-eIF4E (S209), S6 (54D2), p-S6 (S235/236), 
AKT (9272), p-AKT (S473), ERK1/2 (9102), p-ERK1/2 (T202/Y204), p38 (9212), p-p38 (T180/Y182), cleaved caspase-3 (Asp175), 4E-BP1 (53H11), and P-4E-BP1 (T37/46, S65, and T70) were obtained from Cell Signaling. The antibody against $\alpha$-tubulin was generated in house and used as a hybridoma supernatant, whereas Ki67 antibody was from Thermo Scientific. Cells were homogenized in lysis buffer $(50 \mathrm{mM}$ Tris-HCl, pH 7.5, $120 \mathrm{mM}$ $\mathrm{NaCl}, 1 \% \mathrm{NP}-40,1 \mu \mathrm{g} / \mathrm{ml}$ leupeptin, $1 \mu \mathrm{g} / \mathrm{ml}$ aprotinin, $1 \mathrm{mM}$ PMSF, $1 \mathrm{mM}$ benzamidine, $1 \mathrm{mM}$ sodium orthovanadate, $1 \mathrm{mM} \mathrm{NaF}$ ). Aliquots of $50-\mu \mathrm{g}$ protein extracts were separated by SDS-PAGE and transferred to PVDF membranes (Millipore) by electroblotting. Membranes were blocked with $5 \%$ BSA in TBST $(0.1 \%$ Tween 20$)$ and incubated overnight with the primary antibody, followed by a 1-hour incubation with HRP-conjugated secondary antibody. Protein-specific signals were detected using an enhanced chemiluminescence reagent and analyzed using ImageJ software (NIH) (52).

Isolation of initiation complexes using $m^{7}$ GTP and immunoprecipitation. Treated SILAC-labeled and control unlabeled cells were lysed in 0.5\% NP-40 buffer supplemented with protease and phosphatase inhibitors (as described above), and cell debris was removed by centrifugation. Appropriate heavy- and light-labeled samples were combined, precleared with sepharose, and used for precipitation of translation initiation complexes using 7-methyl-GTP-sepharose (GE) for 4 hours at room temperature. A negative control for unspecific binding was prepared using sepharose only. For immunoprecipitation experiments, whole protein cell lysates from treated cells were prepared as described above, precleared with protein G-sepharose (GE), and incubated with a 4EBP1-specific antibody (Cell Signaling) and $20 \mu \mathrm{l}$ of protein G-sepharose (GE) for 4 hours at room temperature. After 5 washes with NP-40 buffer, elution was accomplished with $100 \mu$ l of $0.2 \mathrm{M}$ glycine, $\mathrm{pH} 2.5$, and neutralized with $1.5 \mathrm{M}$ Tris, $\mathrm{pH}$ 8.8. TCA-precipitated, acetone-washed, and air-dried pellets were subjected to mass spectrometry.

Mass spectrometry. The protein pellets were dissolved in RCM buffer (0.5 M Tris, $\mathrm{pH} 8.6,6 \mathrm{M} \mathrm{GnHCl}$ ), and reduction and alkylation steps were accomplished in $20 \mathrm{mM}$ TCEP and $40 \mathrm{mM}$ iodoacetamide, respectively. The proteins were cleaved with trypsin (Promega) or with trypsin followed by Asp-N (Roche) after 6-fold dilution of the samples in $50 \mathrm{mM}$ Tris and $5 \mathrm{mM} \mathrm{CaCl}_{2}(\mathrm{pH} 7.4)$ at $37^{\circ} \mathrm{C}$ overnight. The peptides generated were separated on a $75 \mu \mathrm{m} \times 10 \mathrm{~cm}$ Magic C18 column (Michrom, BioResources) with an Agilent 1100 Nanoflow LC System (Agilent), as described previously (53). The LC was connected to an LTQ Orbitrap Velos (Thermo Scientific), and the data were acquired using 60,000 resolution for the peptide measurements in the Orbitrap and a top-20 method with CID fragmentation and fragment measurement in the LTQ, according to the recommendations of the manufacturer. Data were analyzed using Proteome Discoverer (Thermo Scientific) and Mascot (Matrix Science). The search parameters were tryptic and/or AspN digestion, maximum of 2 missed cleavages, fixed carboxyamidomethyl modifications of cysteine, variable oxidation modifications of methionine, and variable phosphorylation of Ser, Thr, and Tyr, respectively. SILAC samples were also searched with arginine and lysine isotopes as variable modifications. Mass tolerance for precursor ions was $5 \mathrm{ppm}$ and $0.6 \mathrm{Da}$ for fragment ions. Relative quantification was performed with Proteome Discoverer software (Thermo Scientific).

Cell proliferation and apoptosis. Cells were seeded in 6-well plates before transfection and/or treatment, and the number of viable cells was measured using the CellTiter 96 AQueous Non-Radioactive Cell Proliferation Assay (Promega) according to the manufacturer's instructions. Absorbance of MTS bioreduced by cells into a formazan was measured at $570 \mathrm{~nm}$ with a reference of $650 \mathrm{~nm}$.

To analyze numbers of apoptotic cells, treated and control cells were harvested by trypsinization, washed 3 times in PBS, resuspended in $1 \mathrm{x}$ binding buffer, and stained with APC Annexin V (Biolegend) and 7-ADD (Becton
Dickinson). Fluorescence was measured for up to 15,000 cells using a BD FACScan cytometer and analyzed using BD FACSDiva software.

In vivo experiments. U87MG-luc cells were grown under the standard conditions described above in the presence of G418 (Gibco, $50 \mu \mathrm{g} / \mathrm{ml}$ ). Prior to implantation, cells were harvested and resuspended in sterile PBS at $5 \times 10^{6}$ cells per $\mathrm{ml}$. Immunocompromised nude mice (Harlan, Hsd:Athymic Nude-Foxn $1^{n u}$ ) were housed in IVC cages under sterile conditions. All experiments were performed on 6- to 10-week-old female knockout mice in accordance with Swiss Animal Protection Laws. The mice were anesthetized by i.p. injection of a ketamine/xylazine/acepromazine (80:15:5 mg/kg body weight) cocktail. For implantation, the scalp was incised $(\sim 0.7 \mathrm{~cm})$, and the animals were placed in a stereotaxic frame (Harvard Apparatus). A hole was drilled $2 \mathrm{~mm}$ caudal and $2 \mathrm{~mm}$ lateral (right) of the bregma with a dental drill. A $10-\mu 1$ aliquot of the tumor cell suspension (total $5 \times 10^{5}$ cells per animal) was injected $3 \mathrm{~mm}$ below the dura mater over a period of 2 minutes using a 27 -gauge disposable needle on a $100-\mu 1$ syringe (Hamilton) controlled by a motorized injector (Stoelting). The skin was closed with surgical clips. After implantation and 7-day recovery, tumors were measured and animals were stratified into experimental groups. CGP57380 (25 mg/kg) and/or RAD001 (3 mg/kg) in PBS were administered via i.p. injection. During treatment cycles no signs of acute toxicity were observed in any of the groups. At termination, no significant differences in body or organ weight, general health, or anatomy were observed. BLI was performed twice a week, and values were normalized to initial tumor size. In this in vivo model, BLI values correlate with MRI-based tumor size assessment (54). For BLI, the animals received i.p. injections $(10 \mu \mathrm{l} / \mathrm{g}$ body weight) of luciferin (Caliper Life Sciences, D-Luciferin Firefly, potassium salt $)$ in PBS $(15 \mathrm{mg} / \mathrm{ml})$. The animals were anesthetized by isoflurane inhalation ( $3 \%$ induction, $1.5 \%$ maintenance), and tumors were imaged 10 minutes after luciferin administration in a bioluminescence imager (Bertold, NightOWL). Peak emission was measured at $560 \mathrm{~nm}$.

IHC. Paraffin sections of formalin-fixed human tissues were obtained from the University of Basel in accordance with guidelines of the Ethical Committee of the University Hospitals of Basel. Tumors were diagnosed and graded according to the current WHO Classification of Tumors of the Nervous System (55). Brain tissue obtained during surgery for nonlesional epilepsy was used as control. IHC was performed using monoclonal antibodies from Cell Signaling, unless otherwise indicated. Anti-4EBP1 or anti-4EBP1-S65 antibodies were used at 1:1,000 or 1:50 dilutions, respectively. For detection, deparaffinized and rehydrated slides were pretreated in $10 \mathrm{mM}$ citrate buffer, $\mathrm{pH} 6.0$, at $98^{\circ} \mathrm{C}$ for 60 minutes, followed by incubation with $4 \%$ fat-free milk in PBS for 90 minutes. For avidin/biotin blocker treatment (Invitrogen) and detection, the $\mathrm{ABC}$ method was used according to the manufacturer's instructions. P-MNK1-T197/202, p-S6-S235/236, and p-eIF4E-S209 (Abcam, EP2151Y antibody); Ki67 (Thermo Scientific, SP6); cleaved caspase-3, R132H-IDH1 (Dianova, H09); PTEN (Leica, 28H6); and p53 (Dako, DO-7) specific signals were recorded in GBM samples using an automated instrument reagent system (Discovery XT, Ventana Medical System Inc.) according to the user manual. Images of hematoxylin-counterstained sections were captured (Nikon, YTHM) and analyzed using ImageAccess Enterprise7 and Image software. The extent of 4EBP1 or 4EBP1-S65 staining was scored semiquantitatively by a board-certified neuropathologist (S. Frank).

Statistics. $P$ values were obtained by 2 -tailed Student's $t$ tests, and values of $P<0.05$ were considered statistically significant. In animal experiments, the statistical significance of growth curves was calculated as described previously $(56,57)$. Pearson's correlation coefficient was used to determine possible association among signal levels in the analyzed tumors. 
Study approval. All animal procedures were conducted with the approval of the veterinary office of Basel, Switzerland (Veterinaeramt des Kantons Basel-Stadt).

\section{Acknowledgments}

We would like to thank Petra Hirschmann (Institute of Pathology, University of Basel) and Sandrine Bichet (Friedrich Miescher Institute) for help with IHC and Patrick King for editing the manuscript. We thank Jan Seebacher (Friedrich Miescher Institute) for stimulating discussions on proteomics data analysis. This research was funded by the Swiss Cancer League (KFS 2714-08-2010 to B.A. Hemmings and M. Grzmil) and supported by the Novartis Research Foundation.

1. Cancer Genome Atlas Research Network. Comprehensive genomic characterization defines human glioblastoma genes and core pathways. Nature. 2008; 455(7216):1061-1068.

2. Akhavan D, Cloughesy TF, Mischel PS. mTOR signaling in glioblastoma: lessons learned from bench to bedside. Neuro Oncol. 2010;12(8):882-889.

3. Houchens DP, Ovejera AA, Riblet SM, Slagel DE. Human brain tumor xenografts in nude mice as a chemotherapy model. Eur J Cancer Clin Oncol. 1983; 19(6):799-805.

4. Chang SM, et al. Phase II study of CCI-779 in patients with recurrent glioblastoma multiforme. Invest New Drugs. 2005;23(4):357-361.

5. Galanis E, et al. Phase II trial of temsirolimus (CCI-779) in recurrent glioblastoma multiforme: a North Central Cancer Treatment Group Study. J Clin Oncol. 2005;23(23):5294-5304.

6. Sonenberg N, Hinnebusch AG. Regulation of translation initiation in eukaryotes: mechanisms and biological targets. Cell. 2009;136(4):731-745.

7. Silvera D, Formenti SC, Schneider RJ. Translational control in cancer. Nat Rev Cancer. 2010; 10(4):254-266.

8. Grzmil M, Hemmings BA. Translation regulation as a therapeutic target in cancer. Cancer Res. 2012;72(16):3891-3900.

9. Smith MR, et al. Translation initiation factors induce DNA synthesis and transform NIH 3T3 cells. New Biol. 1990;2(7):648-654.

10. De Benedetti A, GraffJR. eIF-4E expression and its role in malignancies and metastases. Oncogene. 2004; 23(18):3189-3199.

11. Buxade M, Parra-Palau JL, Proud CG. The Mnks: MAP kinase-interacting kinases (MAP kinase signal-integrating kinases). Front Biosci. 2008;13:5359-5373.

12. Wendel HG, et al. Dissecting eIF4E action in tumorigenesis. Genes Dev. 2007;21(24):3232-3237.

13. Fan S, Ramalingam SS, Kauh J, Xu Z, Khuri FR, Sun SY. Phosphorylated eukaryotic translation initiation factor 4 (eIF4E) is elevated in human cancer tissues. Cancer Biol Ther. 2009; 8(15):1463-1469.

14. Topisirovic I, Ruiz-Gutierrez M, Borden KL. Phosphorylation of the eukaryotic translation initiation factor eIF4E contributes to its transformation and mRNA transport activities. Cancer Res. 2004; 64(23):8639-8642.

15. Phillips A, Blaydes JP. MNK1 and EIF4E are downstream effectors of MEKs in the regulation of the nuclear export of HDM2 mRNA. Oncogene. 2008; 27(11):1645-1649.

16. Bianchini A, et al. Phosphorylation of eIF4E by MNKs supports protein synthesis, cell cycle progression and proliferation in prostate cancer cells. Carcinogenesis. 2008;29(12):2279-2288.

17. Furic L, et al. eIF4E phosphorylation promotes tumorigenesis and is associated with prostate cancer progression. Proc Natl Acad Sci U S A. 2010; 107(32):14134-14139.

18. Ueda $\mathrm{T}$, et al. Combined deficiency for MAP kinase-interacting kinase 1 and 2 (Mnk1 and Mnk2) delays tumor development. Proc Natl Acad SciU S A. 2010;107(32):13984-13990.

19. Grzmil M, et al. MAP kinase-interacting kinase 1 regulates SMAD2-dependent TGF-beta signaling pathway in human glioblastoma. Cancer Res. 2011; 71(6):2392-2402.

20. Knauf U, Tschopp C, Gram H. Negative regulation of protein translation by mitogen-activated protein kinase-interacting kinases 1 and 2. Mol Cell Biol. 2001;21(16):5500-5511.

21. Bain J, et al. The selectivity of protein kinase inhibitors: a further update. Biochem J. 2007; 408(3):297-315.

22. Liu $Q$, et al. Discovery of 9-(6-aminopyridin-3-yl)-1-(3-(trifluoromethyl)phenyl)benzo[h] $[1,6]$ naphthyridin-2(1H)-one (Torin2) as a potent, selective, and orally available mammalian target of rapamycin (mTOR) inhibitor for treatment of cancer. J Med Chem. 2011;54(5):1473-1480.

23. Thoreen CC, Chantranupong L, Keys HR, Wang T, Gray NS, Sabatini DM. A unifying model for mTORC1-mediated regulation of mRNA translation. Nature. 2012;485(7396):109-113.

24. Rubin JB, et al. A small-molecule antagonist of CXCR4 inhibits intracranial growth of primary brain tumors. Proc Natl Acad Sci U S A. 2003; 100(23):13513-13518.

25. Sanson $\mathrm{M}$, et al. Isocitrate dehydrogenase 1 codon 132 mutation is an important prognostic biomarker in gliomas. J Clin Oncol. 2009;27(25):4150-4154.

26. Engelman JA, et al. Effective use of PI3K and MEK inhibitors to treat mutant Kras G12D and PIK3CA H1047R murine lung cancers. Nat Med. 2008; 14(12):1351-1356.

27. Robinson JP, Vanbrocklin MW, Lastwika KJ, McKinney AJ, Brandner S, Holmen SL. Activated MEK cooperates with Ink4a/Arf loss or Akt activation to induce gliomas in vivo. Oncogene. 2011; 30(11):1341-1350.

28. She QB, Solit DB, Ye Q, O’Reilly KE, Lobo J, Rosen $\mathrm{N}$. The BAD protein integrates survival signaling by EGFR/MAPK and PI3K/Akt kinase pathways in PTEN-deficient tumor cells. Cancer Cell. 2005; $8(4): 287-297$

29. Wang $X$, et al. Inhibition of mammalian target of rapamycin induces phosphatidylinositol 3-kinase-dependent and Mnk-mediated eukaryotic translation initiation factor $4 \mathrm{E}$ phosphorylation. Mol Cell Biol. 2007;27(21):7405-7413.

30. Marzec M, Liu X, Wysocka M, Rook AH, Odum N, Wasik MA. Simultaneous inhibition of mTORcontaining complex 1 (mTORC1) and MNK induces apoptosis of cutaneous T-cell lymphoma (CTCL) cells. PLoS One. 2011;6(9):e24849.

31. She QB, et al. 4E-BP1 is a key effector of the oncogenic activation of the AKT and ERK signaling pathways that integrates their function in tumors. Cancer Cell. 2010;18(1):39-51.

32. Armengol G, et al. 4E-binding protein 1: a key molecular "funnel factor" in human cancer with clinical implications. Cancer Res. 2007;67(16):7551-7555.

33. Rojo F, et al. 4E-binding protein 1 , a cell signaling hallmark in breast cancer that correlates with pathologic grade and prognosis. Clin Cancer Res. 2007; 13(1):81-89.

34. O'Reilly KE, et al. Phosphorylated 4E-BP1 is associated with poor survival in melanoma. Clin Cancer Res. 2009;15(8):2872-2878.

35. Heesom KJ, Gampel A, Mellor H, Denton RM. Cell cycle-dependent phosphorylation of the translational repressor eIF-4E binding protein-1 (4E-BP1). Curr Biol. 2001;11(17):1374-1379.

36. Rannou Y, et al. MNK1 kinase activity is required for abscission. J Cell Sci. 2012;125(pt 12):2844-2852.

37. Gingras AC, et al. Regulation of 4E-BP1 phosphorylation: a novel two-step mechanism. Genes Dev. 1999; 13(11):1422-1437.

38. Gingras AC, et al. Hierarchical phosphorylation of the translation inhibitor 4E-BP1. Genes Dev. 2001; 15(21):2852-2864.

39. Wang X, Li W, Parra JL, Beugnet A, Proud CG. The $\mathrm{C}$ terminus of initiation factor $4 \mathrm{E}$-binding protein 1 contains multiple regulatory features that influence its function and phosphorylation. Mol Cell Biol. 2003;23(5):1546-1557.

40. Proud CG. Signalling to translation: how signal transduction pathways control the protein synthetic machinery. Biochem J. 2007;403(2):217-234.

41. Waskiewicz AJ, Flynn A, Proud CG, Cooper JA. Mitogen-activated protein kinases activate the serine/ threonine kinases Mnk1 and Mnk2. EMBO J. 1997; 16(8):1909-1920.

42. Fukunaga R, Hunter T. MNK1, a new MAP kinase-activated protein kinase, isolated by a novel expression screening method for identifying protein kinase substrates. EMBOJ. 1997;16(8):1921-1933.

43. Carracedo A, et al. Inhibition of mTORC1 leads to MAPK pathway activation through a PI3K-dependent feedback loop in human cancer. J Clin Invest. 2008;118(9):3065-3074.

44. Ueda T, Watanabe-Fukunaga R, Fukuyama $H$, Nagata S, Fukunaga R. Mnk2 and Mnk1 are essential for constitutive and inducible phosphorylation of eukaryotic initiation factor $4 \mathrm{E}$ but not for cell growth or development. Mol Cell Biol. 2004; 24(15):6539-6549.

45. Astanehe A, et al. MKNK1 is a YB-1 target gene responsible for imparting trastuzumab resistance and can be blocked by RSK inhibition. Oncogene. 2012;31(41):4434-4446.

46. Chrestensen CA, et al. Loss of MNK function sensitizes fibroblasts to serum-withdrawal induced apoptosis. Genes Cells. 2007;12(10):1133-1140.

47. Hu SI, et al. MNK2 inhibits eIF4G activation through a pathway involving serine-arginine-rich protein kinase in skeletal muscle. Sci Signal. 2012; 5(211):ra14.

48. Adesso L, et al. Gemcitabine triggers a pro-survival response in pancreatic cancer cells through activation of the MNK2/eIF4E pathway. Oncogene. 2013; 32(23):2848-2857.

49. Shi Y, et al. MNK kinases facilitate c-myc IRES 
activity in rapamycin-treated multiple myeloma cells. Oncogene. 2013;32(2):190-197.

50. Huo Y, et al. Stable isotope-labelling analysis of the impact of inhibition of the mammalian target of rapamycin on protein synthesis. Biochem J. 2012; 444(1):141-151.

51. Ilic N, Utermark T, Widlund HR, Roberts TM. PI3K-targeted therapy can be evaded by gene amplification along the MYC-eukaryotic translation initiation factor 4E (eIF4E) axis. Proc Natl Acad Sci U S A. 2011;108(37):E699-E708.

52. Schneider CA, Rasband WS, Eliceiri KW. NIH
Image to ImageJ: 25 years of image analysis Nat Methods. 2012;9(7):671-675.

53. Hess D, Keusch JJ, Oberstein SA, Hennekam RC, Hofsteenge J. Peters Plus syndrome is a new congenital disorder of glycosylation and involves defective Omicron-glycosylation of thrombospondin type 1 repeats. J Biol Chem. 2008;283(12):7354-7360.

54. Szentirmai O, et al. Noninvasive bioluminescence imaging of luciferase expressing intracranial U87 xenografts: correlation with magnetic resonance imaging determined tumor volume and longitudinal use in assessing tumor growth and antiangiogenic treatment effect. Neurosurgery. 2006;58(2):365-372

55. Louis DN, et al. The 2007 WHO classification of tumours of the central nervous system. Acta Neuropathol. 2007;114(2):97-109.

56. Elso CM, et al. Leishmaniasis host response loci (lmr1-3) modify disease severity through a Th1/ Th2-independent pathway. Genes Immun. 2004; 5(2):93-100.

57. Baldwin $\mathrm{T}$, et al. Wound healing response is a major contributor to the severity of cutaneous leishmaniasis in the ear model of infection. Parasite Immunol. 2007; 29(10):501-513 